\title{
Analysis of Oscillatory Processes in Elastic and Viscoelastic Media and Structural Elements to Predict Equipment Shutdown
}

\author{
E. Mailybayev ${ }^{1}$, U. Umbetov $^{2 *}$, A. Seitmuratov ${ }^{3}$, A. Shynykulova ${ }^{1}$ \\ ${ }^{1}$ Kazakh University Ways and Communications, 32a, mkr. Zhetysu-1, Almaty, 050063, Republic of Kazakhstan; \\ ${ }^{2}$ Kh.A. Yasavi International Kazakh-Turkish University, 29, prosp. B. Sattarkhanova, Turkestan, Republic of Kazakhstan; \\ ${ }^{3}$ Korkyt ata Kyzylorda State University, 29A, Ayteke Bi ul., Kyzylorda, Republic of Kazakhstan \\ * Corresponding author. Tel.: 8 (72533) 638 48.E-mail: uumbetov@mail.ru
}

\begin{abstract}
Free vibration problems of a flat element are investigated by all boundary value problems; generalization of the decomposition method in dynamics is given to solve boundary value problems, while it is shown that the decomposition method gives an exact solution obtained by the direct method, which in turn makes it possible to test the parts of production plants for wear leading to the shutdown of the equipment or the whole process. By checking the strength of the equipment parts, studying the degree of risk of possible breakdowns, emergency shutdowns can be predicted, and it is also possible to create controlled shutdowns.
\end{abstract}

\section{Keywords}

Oscillatory process; free vibration problems; decomposition method; equipment shutdown prediction; elastic and viscoelastic media.

\section{Introduction}

The development of science and technology, the creation of new structures, the use of high-quality materials and technologies that meet the high level of scientific and technological progress, puts forward high demands on research in the field of dynamics of deformable media.

Applied problems and the laws of the internal development of basic research in mechanics of a deformable solid revealed tendencies to sequentially take into account physical and mechanical properties of materials, the nature of their deformation in time, the effects of the relationship of mechanical deformation fields with temperature, electric and magnetic fields, and the geometric structure of bodies. Among these problems one of the leading places is occupied by the problems of theoretical analysis of oscillatory processes in elastic and viscoelastic media and structural elements that non-stationary interact with the surrounding deformable medium.

The study is the subject of the general theory of oscillation and the theory of waves, which are now widely developed.
Positive results of these studies are useful when considering stationary, non-stationary oscillatory and wave processes in the operation of production equipment with the aim of monitoring wear of parts and pre-warning of possible shutdowns. Fig. 1 shows top view of boundless plate with thickness $2 h_{1}$.

In modern industrial equipment materials with viscoelastic properties, in particular, polymeric ones are becoming more widespread, fundamental research in the field of unsteady processes of deformation of viscoelastic bodies and specific calculations of structural elements of viscoelastic materials are widely used in various fields of engineering practice. This includes the problems of determining strength, evaluating reliability and durability, determining frequency characteristics, choosing optimal parameters that provide effective operating conditions, stability, and some other issues related to the behavior of structural elements when exposed to dynamic effects.

These studies have a wide range of relevant applications in such fields of science and technology as seismology, geophysics, acoustic flaw detection, mechanical engineering and space technology [1]. 


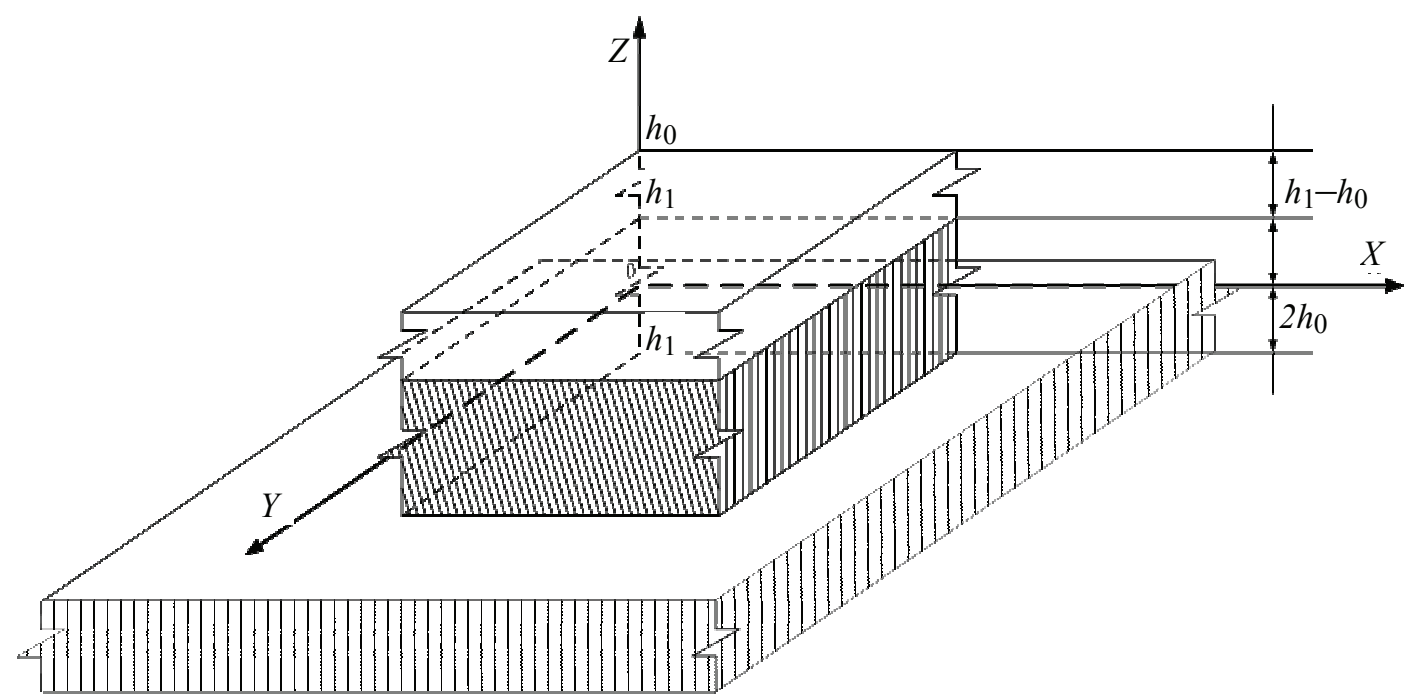

Fig. 1. Boundless plate with thickness $2 h_{1}$ lying under the upper layer $h_{0}-h_{1}$ and on the surface of half-space $z<-h_{1}$

The urgent problem of the current stage of theoretical research in the field of unsteady oscillations of viscoelastic bodies, along with the development of new models of dynamic deformation of viscoelastic materials close to experimental, is the development of effective mathematical methods for studying many classes of plane and spatial problems within the framework of known models, theoretical analysis of the main mechanical factors caused by the effect of viscoelastic parameters.

Despite the large number of theoretical and applied research in this field, the problems of solving many important classes of boundary value problems and their analysis remain mostly open or require further refined development. These include the problems of unsteady oscillations of roads, plates and shells, taking into account rheology.

When solving problems of this class, approximate oscillation equations obtained from the threedimensional theory of equations of motion of the theory of elasticity using various hypotheses and assumptions of a mechanical or geometric nature that significantly simplify the solution of the problem are used as the main resolving equations. In addition, the original three-dimensional problem of the theory of elasticity is reduced to two-dimensional or one-dimensional using various mathematical methods, including variational and asymptotic methods, the power series method, etc.

At present, a large number of studies have been carried out to reduce the three-dimensional problem to two-dimensional engineering and mathematical methods. But these studies do not solve the problem completely: therefore, the problem of studying the dynamic behavior of circular rods interacting with a deformable medium on the basis of vibration equations derived using a rigorous mathematical apparatus requires further investigation. The scope of such problems is wide enough, because circular rods are elements of many engineering structures, ranging from the simplest machines, instruments and structures, to the most sophisticated space technology, nuclear and hydroelectric power plants, shipbuilding, etc.

Taking into account the rheological properties and anisotropy of the material, the shell of the interacting medium, changes in temperature, thickness variability and other factors lead to a significant complication in the study of these problems. On the other hand, the correct consideration of these factors is of great importance for ensuring the strength, reliability and durability of structures, which can significantly save material resources and minimize equipment wear [2].

Wear is a gradual surface destruction of the material with a change in the geometric shapes and properties of the surface layers of the parts. Wear can be normal and abnormal.

Depending on the reasons, wear is divided into 3 categories - chemical, physical and thermal.

Normal wear involves short-term dimensional changes due to improper installation, operation and maintenance. Chemical wear involves the formation of parts of the thinnest oxide layers with subsequent exfoliation of these layers on the surface. The occurring destruction is accompanied by the appearance of rust, metal corrosion. Physical wear is caused by excessive loads, surface friction, abrasive and mechanical stress.

At the same time, the parts might develop microcracks or cracks, or the surface of the metal might become rough. 
Physical wear can come in different forms: confluent pitting, fatigue, abrasive erosion, erosion.

Thermal wear is characterized by the appearance and subsequent destruction of molecular bonds inside the metal. It is caused by increased or decreased temperature. The reasons affecting wear are as follows:

1. Quality of material of parts.

As a rule, for most parts the wear resistance is higher, the harder their surface but not always the degree of hardness is directly proportional to the wear resistance.

Materials with only high hardness have high wear resistance.However, this increases the likelihood of risk and detachment of particles of the material. Therefore, such parts must have a high viscosity, which prevents the separation of particles. If two parts from homogeneous materials are subject to friction, then with an increase in the coefficient of friction they quickly wear out, therefore more expensive and difficult to replace parts need to be made of harder, better and more expensive material, and cheaper simple parts should be made of material with a low coefficient of friction.

2. Quality of the surface treatment of the part.

Three periods of wear of the part have been established:

- the initial wear is characterized by a rapid increase in the gap of movable joints;

- the period of steady wear is-characterized by slow, gradual wear;

- he period of rapid, increasing wear is caused by a significant increase in gaps and change in the geometric shapes of parts.

To increase the service life of parts it is necessary:

- to reduce the first period as much as possible, by means of very precise and clean processing of parts;

- to maximize the second period;

- to prevent a third period.

3. Lubrication.

A layer of grease introduced between the rubbing parts fills all the roughness and unevenness and reduces friction and wear by many times.

4. Speed of movement of parts and specific pressure.

Based on experimental data, it was found that at normal specific loads and speeds from 0.05 to 0.7 the oil layer does not break and the part works for a long time. If you increase the load, the wear of the part increases many times. [3].

5. Infringement of rigidity in motionless details.

6. Violation of fit.

7. Violation of the relative position of the parts in the joints.
Today, most equipment used at enterprises has automated control systems for monitoring of process parameters. Such systems make it possible to collect process parameters, store data on equipment operating modes, and notify about emergency situations and malfunctions. The relevance of developing methods for determining the state of equipment based on process parameters is explained by the fundamental need for a modern determination of the conditions of machines and assemblies [4].

During the operation of ASM various accidents can occur - sensor malfunction, communication line breakdown, controller/computer failure, output of those process parameters beyond the established boundaries. In order to ensure the continuity of the process, the control system must be equipped with means for detecting and processing emergency situations: the following tools are provided in the Tracemode 6 for this purpose:

- automatic installation of a sign of hardware unreliability to a channel associated with Input/Output equipment or a channel of another node via any of the available interaction interfaces in the event of a failure, data exchange;

- automatic installation of a sign of program unreliability to the channel when its value goes beyond the set limits;

- monitoring the value of FLOAT channels (analog alarms) - for these channels 6 boundaries are set with the help of which timely detection of an abnormal state of the process is possible;

- monitoring events (including accidents) using the event class channel;

The Trace Mode 6 has the means to perform actions aimed at preventing the occurrence or development of an accident during operation of ASM. Such means include, for example, the ability to turn on an alarm, to issue recommendations to the operator, blocking. Process status information can be stored in archives and in the alarm report.

\section{Experimental}

For the numerical analysis of the problems of oscillatory processes in elastic and viscoelastic media, one can successfully apply the approximate method of obtaining frequency equations based on the decomposition method developed in the works of professor G. Pshenichny [5, 6] for static problems. We consider a number of problems of oscillations of flat rectangular elements under arbitrary boundary conditions as the edges of the element in order to determine the frequencies of natural oscillations by the decomposition method. We state the statement of the 


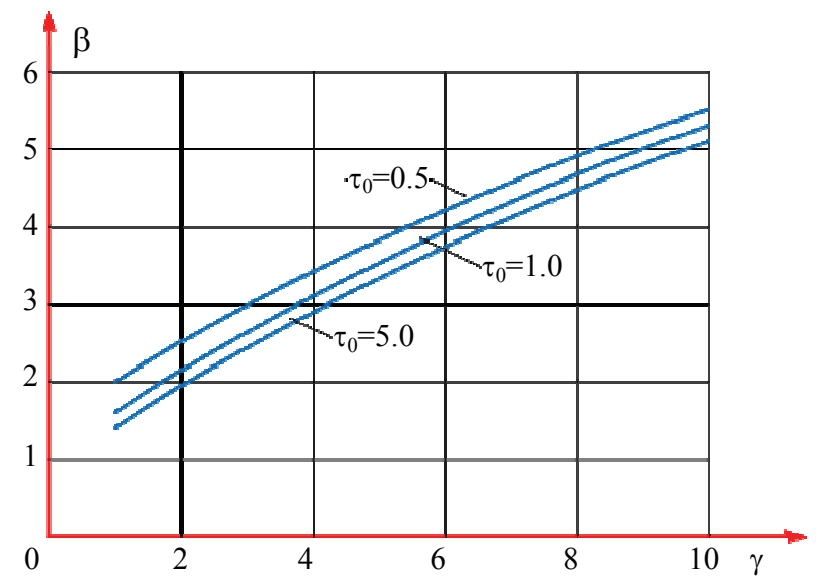

Fig. 2. Curves of changes in the frequencies of natural vibrations for a viscoelastic plate at $\tau_{0}=0.5, \tau_{0}=1.0, \tau_{0}=5.0, v_{1}=0.34, v_{2}=0.3$

method for the case of a flat element, when the material of the element is elastic. In the future, the method will be applied to elements of viscoelastic material.

Fig. 2 shows the curves of changes in the frequencies of natural vibrations for a viscoelastic plate.

In the case of a flat part made of elastic material we write the approximate equation of the transverse vibration of the fourth order in the form

$$
\Delta^{2} W-D_{0} \frac{\partial^{2}}{\partial t^{2}} \Delta W+D_{1} \frac{\partial^{4} W}{\partial t^{4}}+D_{2} \frac{\partial^{2} W}{\partial t^{2}}=0,
$$

where the coefficients $D_{0}, D_{1}, D_{2}$ are determined by the geometry and properties of the material of the flat element.

We will look for a solution to the equation in the form (1)

$$
W=\exp \left(\begin{array}{c}
b \\
i- \\
h
\end{array}\right) W_{0}(x, y) .
$$

Substituting (2) for $W_{0}$ we obtain the equation

$$
\begin{gathered}
\Delta^{2} W_{0}-D_{0}\left(\frac{b}{h}\right)^{2} \xi^{2} \Delta W_{0}+ \\
+\xi^{2}\left(\frac{b}{h}\right)^{2}\left[D_{1}\left(\frac{b}{h}\right)^{2} \xi^{2}-D_{2}\right] W_{0}=0 .
\end{gathered}
$$

For the decomposition method it is more convenient to introduce new independent and dependent variables

$$
\alpha=\frac{\pi}{l_{1}} x ; \beta=\frac{\pi}{l_{2}} y ; \quad W_{0}=\frac{l_{1}^{4}}{\pi^{4}} v ;
$$

$$
\lambda=\frac{l_{1}}{l_{2}} ; \quad \lambda_{1}=\frac{l_{1}}{\beta \pi h} .
$$

In variables (4) the equation (3) takes the form

$$
\begin{gathered}
{\left[\frac{\partial^{4} v}{\partial \alpha^{4}}+2 \lambda^{2} \frac{\partial^{4} v}{\partial \alpha^{2} \partial \beta^{2}}+\lambda^{4} \frac{\partial^{4} v}{\partial \beta^{4}}\right]+\lambda_{1}^{2} D_{0}\left(\frac{b}{h}\right)^{2} \xi^{2} \times} \\
\times\left[\frac{\partial^{2} v}{\partial \alpha^{2}}+\lambda^{2} \frac{\partial^{2} v}{\partial \beta^{2}}\right]+\lambda_{1}^{4}\left(\frac{b}{h}\right)^{2} \xi^{2}\left[D_{1}\left(\frac{b}{h}\right)^{2} \xi^{2}-D_{2}\right] v=0 .
\end{gathered}
$$

The decomposition method in the theory of oscillations in the general setting is as follows [7].

The statement of auxiliary problems is formulated

Problem 1. Find a solution to the equation

$$
\frac{\partial^{4} v_{1}}{\partial \alpha^{4}}=f^{(1)}(\alpha, \beta)
$$

under boundary conditions

$$
L_{1}(\alpha, \beta)=0 ; \quad L_{2}(\alpha, \beta)=0 ; \quad(\alpha=0 ; \pi)=0 .
$$

Problem 2. Find a solution to the equation

$$
\lambda^{4} \frac{\partial^{4} v_{2}}{\partial \beta^{4}}=f^{(2)}(\alpha, \beta)
$$

under boundary conditions

$$
L_{3}(\alpha, \beta)=0 ; \quad L_{4}(\alpha, \beta)=0 ; \quad(\beta=0 ; \pi)=0 .
$$

The boundary conditions at the edges of the plate depend on the conditions of its fastening or on the free edge from stresses.

\section{Results and Discussion}

The remainder of equation (5)

$$
\begin{gathered}
2 \lambda \frac{\partial^{4} v_{3}}{\partial \alpha^{2} \partial \beta^{2}}+\lambda D_{0}\left(\frac{b}{h}\right)^{2} \xi^{2}\left[\frac{\partial^{2} v_{3}}{\partial \alpha^{2}}+\lambda^{2} \frac{\partial^{2} v_{3}}{\partial \beta^{2}}\right]+ \\
+\lambda_{1}^{4} D_{0}\left(\frac{b}{h}\right)^{2} \xi^{2}\left[D_{1}\left(\frac{b}{h}\right)^{2} \xi^{2}-D_{2}\right] v_{3}+ \\
+f^{(1)}(\alpha, \beta)+f^{(2)}(\alpha, \beta)=0,
\end{gathered}
$$

where $f^{(i)}(\alpha, \beta)$ arbitrary functions whose form depends on the boundary value problems being solved.

Following the decomposition method we assume that 


$$
v_{3}=\frac{1}{2}\left[v_{1}+v_{2}\right] .
$$

The condition must also be fulfilled at given points of the plane element.

The general solutions of the equations of auxiliary problems (6) and (8) have the form

$$
\begin{aligned}
& v_{1}=f_{1}(\alpha, \beta)+\frac{\alpha^{3}}{6} \varphi_{1}(\beta)+\frac{\alpha^{2}}{2} \varphi_{2}(\beta)+\alpha \varphi_{3}(\beta)+\varphi_{4}(\beta) ; \\
& v_{1}=f_{1}(\alpha, \beta)+\frac{\beta^{3}}{6} \psi_{1}(\alpha)+\frac{\beta^{2}}{2} \psi_{2}(\alpha)+\beta \psi_{3}(\alpha)+\psi_{4}(\alpha),
\end{aligned}
$$

where $\varphi_{j}, \psi_{j}$ arbitrary functions of the arguments are determined from the boundary conditions (7) and (9).

Then we will represent arbitrary functions in a general form as

$$
f^{(j)}(\alpha, \beta)=\sum_{n=1}^{\infty} \sum_{j=1}^{\infty} a_{n, m}^{(j)} \sin (\alpha n) \sin (\beta m),
$$

where $a_{n, m}^{(j)}$ arbitrary constants, and the functions $f_{j}(\alpha, \beta)$ in the general solutions (12) are equal

$$
\begin{aligned}
& f_{1}(\alpha, \beta)=\sum_{n=1}^{\infty} \sum_{j=1}^{\infty} \frac{a_{n, m}^{(1)}}{n^{4}} \sin (\alpha n) \sin (\beta m) ; \\
& f_{2}(\alpha, \beta)=\sum_{n=1}^{\infty} \sum_{j=1}^{\infty} \frac{a_{n, m}^{(2)}}{m^{4}} \sin (\alpha n) \sin (\beta m) .
\end{aligned}
$$

Using particular solutions to problems under given boundary conditions and using approximate representations (11) to find unknowns $a_{n, m}^{(j)}$ we obtain a homogeneous linear system of algebraic equations, a nontrivial solution of which leads to a frequency equation.

Thus, the approximate decomposition method allows us to find the natural frequencies of flat elements. The problems for the viscoelastic material of a flat element are solved in a similar way.

The basis of automated systems for monitoring the state of equipment laid the concept of a software and hardware complex. The concept of a software and hardware complex appeared relatively recently in the field of computer technology and fiscal devices. One of the popular a software and hardware complex is "Cruise" with Trace Mode software.
A software and hardware complex Cruise is and automated process control system, which is a combination of hardware and software and is designed to implement automatic, automated and remote control of industrial facilities.

Fig. 3 shows the structure of the software and hardware complex Cruise.

The main goals of creating an automated process control system are:

- ensuring the management of technological processes of production in normal, emergency and post-emergency conditions;

- providing operational personnel with sufficient, reliable and timely information about operating modes, the course of technological processes, the condition of equipment and technical controls:

- optimization of technical and economic indicators;

- increasing the reliability of equipment;

- improving working conditions for operating personnel.

Working with the software and hardware complex involves solving the following problems:

- collecting and primary processing of information;

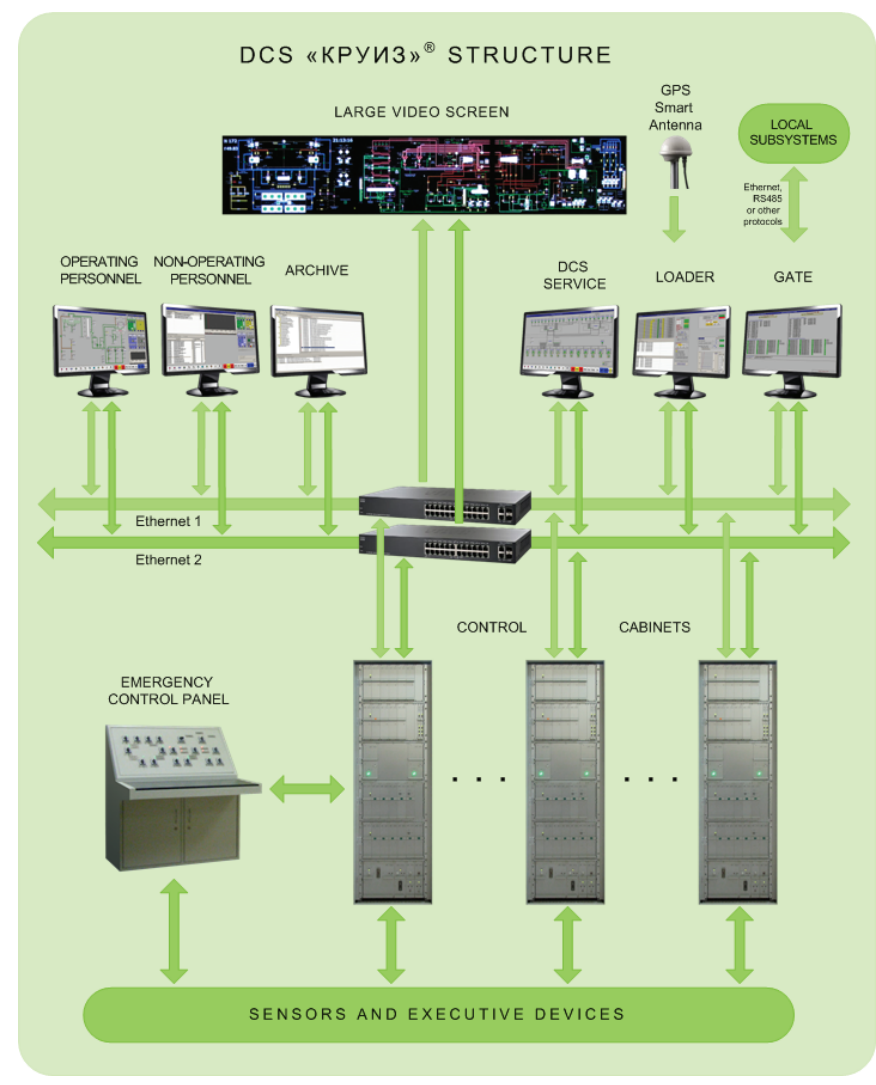

Fig. 3. DCS “Cruise" structure 
- monitoring the reliability of the received information;

- receiving and storing retrospective information;

- creating process equipment monitoring teams; presentation of information to operational and engineering personnel of the process control system in the form of mnemonic, diagrams, graphs, histograms;

- logging and documentation;

- registering emergency events and analysis of protection actions;

- monitoring and displaying the state of the hardware and software complex.

The complex is a distributed control system with a two-level organization, the connection between the various levels of the system is organized on the basis of the standard Ethernet protocol, which allows you to see the vast world experience in building fault-tolerant systems and apply the latest developments in the field of distributed computing and redundancy.

On the whole, the automated control system is a decentralized human-machine system in which part of the control is performed automatically, and partly using remote automated control, i.e., with the participation of an individual. In urgent cases, the process control can be carried out using emergency tools and individual control keys installed on the remote controls of the backup control system. Individual tools of control and monitoring are used to ensure safe shutdown of equipment in the event of a functional failure of the software and hardware complex. For each level of process control systems, corresponding control posts with a permanent stay of operational personnel are provided. Information is generated and displayed by the technical means of the software and hardware complex automatically, while management decisions are made and implemented by the operator. The interaction of the operator with the control system is carried out using the information presentation subsystem [8].

In Trace Mode 6, not only the channel base is automatically created and tuned to the controllers, but also the operator's graphical interface is automatically built using monitors and equipment control algorithms are selected. Fig. 4 shows the recording of emergency stops based on technical parameters in the Trace Mode.

Monitors can generate messages in various situations during the work of an automated control system, for example, when a channel value of the FLOAT class goes beyond a set limit when the employee status changes. These messages are recorded in a special textfile - alarm report (AR) which is configured for the node. AR-messages are entered on channels for which the corresponding flag is set.

Configuration AR enables the monitor to generate messages. Message texts for events can be defined in dictionaries. If the channel is connected to the dictionary, messages from the dictionary are generated; otherwise, the monitor generates messages by default. For some channels, the criteria for generating messages depend on the parameters of these channels.

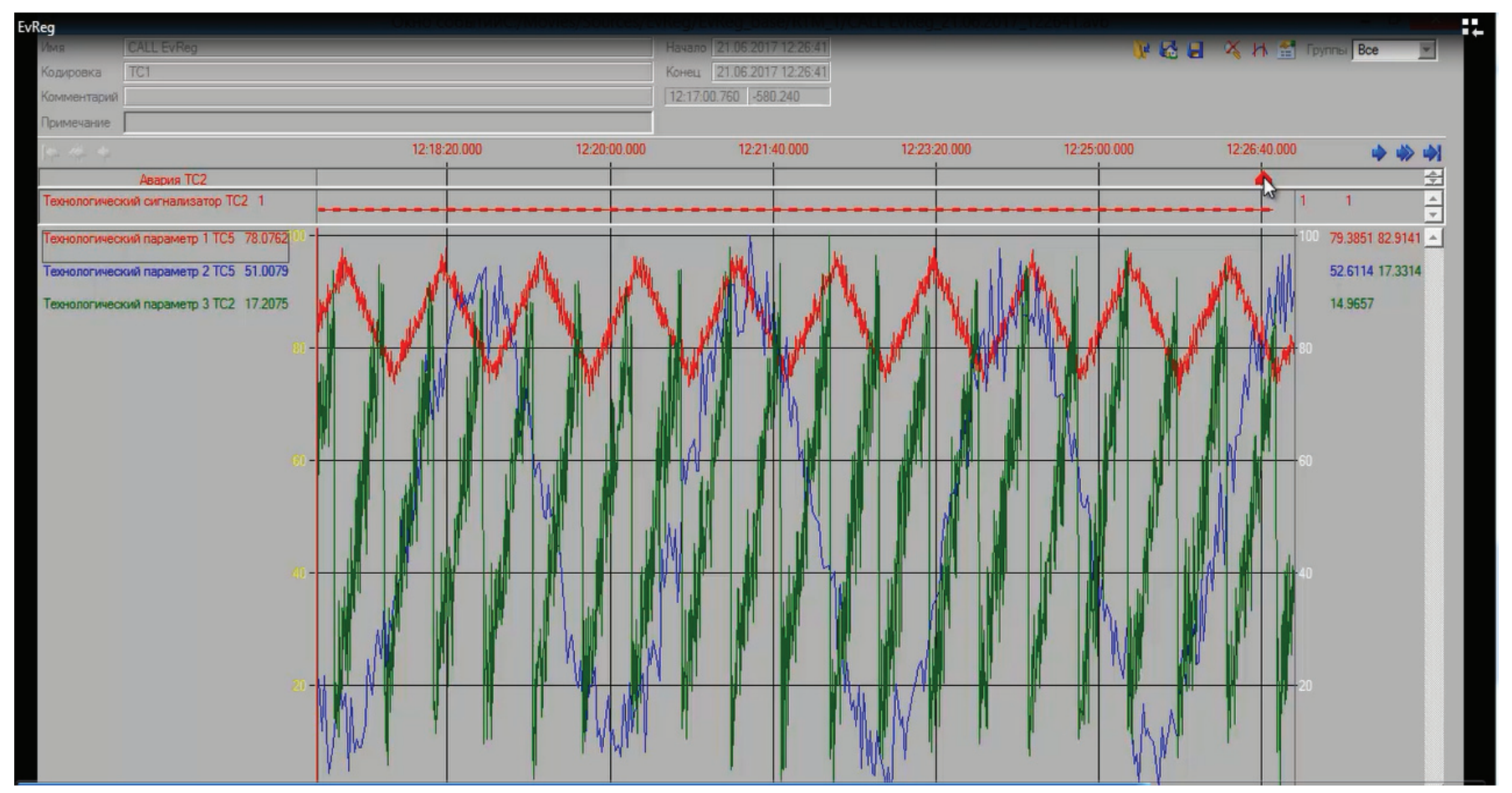

Fig. 4. Registration of emergency stops based on technical parameters in the Trace Mode 


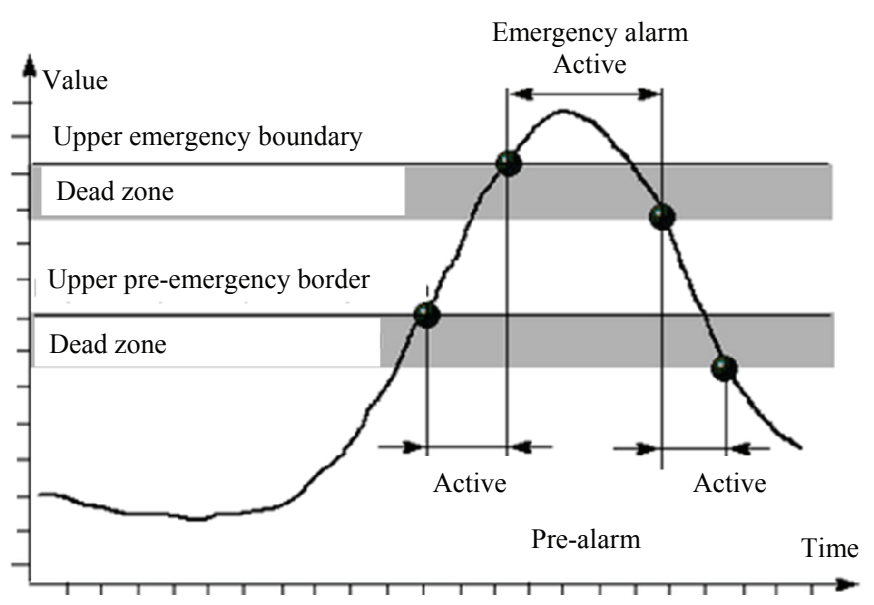

Fig. 5. Alarm combinations

In the dictionary, additional directions for transmitting messages can be set-for example, in the form of SMS messages to a specified cell phone number over a network of consoles. A number of graphic elements used in the development of graphic screens, allows the operator to enter arbitrary messages into the alarm report, as well as view all AR messages and acknowledge them [9].

Alarms are based on the analysis of the output of variable values beyond the specified upper and lower limits. Fig. 5 shows alarms in several combinations:

- upper limits (pre-emergency and emergency);

- lower limits (pre-emergency and emergency);

- deviation from the set value;

- rate of change of the parameter.

To exit a variable from the alarm state, it is necessary that its value becomes less than the threshold by an amount called the deadband. Similarly, lower pre-alarm and emergency alarms can be interpreted. All of the above is true for a deviation type alarm. The set value during the process can be changed either by the operator or by the program. The alarm will be triggered when the value of the variable goes beyond the tolerance limit. Alarms, determined by the rate of change of the parameter occur if it becomes more (less) the maximum permissible. The concept of a "deadband" for alarms of this type does not apply [10].

\section{Conclusion}

The analytical and numerical results obtained in the article on the study of the natural oscillations of planar rectangular elements and the propagation of harmonic waves in planar elements allow us to state the possibility of using the decomposition method to obtain exact solutions of oscillatory processes in elastic and viscoelastic media and structural elements to predict equipment shutdown. Based on the theoretical results, boundary-value problems on the natural vibrations of rectangular plane elements are formulated and solved which are modeled in the Trace Mode program [11].

The problems of free oscillation of a flat element are investigated by all other boundary value problems a generalization of the decomposition method in dynamics is given for solving boundary value problems, and it is shown that the decomposition method gives an exact solution obtained by the direct method, which in turn makes it possible to test the details of production plants for wear leading to shutdown of an individual equipment or a whole technological process.

\section{References}

1. Morokina G. Computer-Aided Design Systems of Decentralization on the Base of Trace Mode in the Industry. International Russian Automation Conference (RusAutoCon), September 2019. doi: 10.1109/rusautocon.2019.8867817

2. Majlybaev E.K., Umbetov U.U., Batyrkanov Zh.I., Kosjakov I.O., Shynykulova A.B. Postroenie otkazoustojchivyh decentralizovannyh sistem [Building Ili Fault-Tolerant Ili Decentralized Ili Systems]. Izvestija KGTU im. I. Razzakova 2019, 2(50), Part 1, 100-104 (Rus)

3. Bondareva G.I. Sovershenstvovanie, nauchnoe obosnovanie $i$ promyshlennoe osnovanie avtomatizirovannyh tehnologicheskih processov povyshenija dolgovechnosti rabochih jelementov tehnologicheskogo oborudovanija: monografija [Improvement, scientific justification and industrial foundation of automated technological processes to increase the durability of the working elements of technological equipment: monograph]. Moscow: Rosinformagroteh, 2010, 242 p. (Rus)

4. Minakov A.A., Fedoseev D.V. Diagnostirovanie oborudovanija s ispol'zovaniem parametrov tehnologicheskogo kontrolja [Diagnostics of equipment using process control parameters]. Izvestija Samarskogo nauchnogo centra Rossijskoj akademii nauk, 2009, 305-309. (Rus)

5. Pshenichnov G.I. Metod dekompozicii reshenija uravnenija i kraevyh zadach [The decomposition method for solving equations and boundary value problems]. DAN SSSR, 1985, 182(4), 792-794. (Rus)

6. Pshenichnov G.I. Reshenija nekotoryh zadach stroitel'noj mehaniki metodom dekompozicii [Solving some problems of structural mechanics by decomposition method]. Stroitel'naja mehanika $i$ raschet sooruzhenij, 1986, 4, 12-17. (Rus)

7. Sejtmuratov A.Zh. Priblizhennyj metod reshenija dinamicheskih zadach dlja linejnyh vjazkouprugih sred [An approximate method for solving dynamic problems for linear viscoelastic media]. Izvestija MGTU Mashinostroenie. 2007, 11, 9-15. (Rus.)

8. DCS Cruise. Full description of the system. Available from: http://pikzebra.ru/ptk/doc/index.php.

9. Gerasimov A.V., Titovcev A.S. SCADA sistema Trace Mode 6 [SCADA System Trace Mode 6]: tutorial. Kazan': Izdatel'stvo Kazan. gos. tehnol. universiteta, 2011, 128 p.(Rus)

10. Anokhina T. Selection and comparison of SCADAsystem. Moscow, 2004.

11. Morokina, G.S., Katsan, I.F., Umbetov, U. Control systems on the base of TM6 in industry. Proceedings of the 31st International Business Information Management Association Conference, IBIMA, 2018, 6566-6570. 